\title{
Prospective Comparison of Redo Microvascular Decompression and Percutaneous Balloon Compression as Primary Surgery for Recurrent Trigeminal Neuralgia
}

\author{
Jing-nan Chen, M.D., ${ }^{1}$ Wen-hua Yu, M.D., ${ }^{2}$ Hang-gen Du, M.D., ${ }^{1}$ Li Jiang, M.D., ${ }^{2}$ Xiao-qiao Dong, M.D., ${ }^{2}$ Jie Cao, M.D. \\ Department of Neurosurgery, The Second Affiliated Hospital of Zhejiang Chinese Medical University, Hangzhou, China \\ Department of Neurosurgery, ${ }^{2}$ The First People's Hospital of Hangzhou, Hangzhou, China
}

Objective : To prospectively compare facial pain outcomes for patients having either a repeat microvascular decompression (MVD) or percutaneous balloon compression (PBC) as their surgery for trigeminal neuralgia (TN) recurrence.

Methods : Prospective cohort study of 110 patients with TN recurrence who had either redo MVD ( $n=68)$ or PBC ( $n=42)$ from July 2010 until September 2016. The mean follow-up was 45.6 months.

Results : After redo MVD, 65 patients (95.6\%) experienced immediate relief of pain. After PBC, 34 patients (81\%) were immediately relieved of their neuralgia. After 1 month, the clinical effect of redo MVD was better than PBC $(p<0.01)$. Patients who had redo MVD more commonly were pain free off medications (93.4\% at 1 year, $78.2 \%$ at 4 years) compared with the PBC patients (85.1\% at 1 year, $59.3 \%$ at 4 years). However, mean length of stay was longer $(p>0.05)$. Patients after PBC who occurred developed herpes simplex (35.7\%), facial numbness (76.2\%), and annoying dysesthesia (21.4\%) more frequently compared with patients after redo MVD who occurred developed herpes simplex (14.7\%), facial numbness (8.8\%), and hypoesthesia (5.9\%) $(p<0.05)$. The symptoms recurred respectively in 15 patients (22.1\%) and 19 patients (45.2\%) after redo MVD and PBC within the entire 6-year follow-up period.

Conclusion : For the patients with TN recurrence, redo MVD was a more effective procedure than PBC. The cure rate and immediate relief of pain were better, and the incidence of complications was lower.

Key Words : Trigeminal neuralgia · Re-do · Microvascular decompression surgery · Percutaneous balloon compression.

\section{INTRODUCTION}

Trigeminal neuralgia (TN) is a disabling disorder characterized by sudden attacks of devastating facial pain which is confined to the distribution of the trigeminal nerve supply. Since microvascular decompression (MVD) has been popularized by
Jannetta in the 1980s, MVD has been the first-line surgical treatment to definitively cure the condition if medical treatment fails to provide sufficient relief ${ }^{10,11,13,17)}$. However, a percentage of patients continue to have TN following MVD over time ${ }^{20,28,30)}$. Recurrent trigeminal neuralgia may be due to insufficient operative techniques, slip of the Teflon implant, insertion of too

- Received : June 24, 2017 • Revised : September 13, 2017 •Accepted : November 24, 2017

- Address for reprints : Wen-hua Yu, M.D.

Department of Neurosurgery, The First People's Hospital of Hangzhou, 261 Huansha Road, Hangzhou 310006, China

Tel : +86-183-6783-5143, Fax : +86-571-87914773, E-mail : Yu19910619@hotmail.com

This is an Open Access article distributed under the terms of the Creative Commons Attribution Non-Commercial License (http://creativecommons.org/licenses/by-nc/4.0) which permits unrestricted non-commercial use, distribution, and reproduction in any medium, provided the original work is properly cited. 
much Teflon, adhesions of the interposed Teflon or in the rare case, development of a granuloma ${ }^{1,7,14,18,22,23,29)}$.

A large number of researches have been carried on MVD postoperative recurrence of trigeminal neuralgia, respectively using repeat MVD and percutaneous balloon compression $(\mathrm{PBC})^{2,8,9,19)}$. Therefore, this study is to compare the curative effect of redo MVD and PBC when first MVD is invalid through a prospective cohort study.

\section{MATERIALS AND METHODS}

\section{Patients}

Since July 2010, the senior author has maintained a prospective database on all patients undergoing surgery for recurrent trigeminal neuralgia. Recurrence was defined as resurgence of typical trigeminal neuralgia pains, requiring reintroduction of medications or any other treatment options, after an initial success in complete pain eradication (requiring no medications for pain) following previous MVD. One hundred and thirteen patients underwent redo MVD or PBC for recurrent TN. Three patients ( $3 \%$ ) had $<6$ months of follow-up and were excluded. All patients were examined by pre-operative magnetic resonance imaging- time of flight (MRI-TOF) brain scan and informed of advantages and disadvantages. Then they made a choice according to their will. The characteristics of the remaining 110 patients (MVD, $n=68$; PBC, $n=42$ ) are outlined in the Table 1. Informed consentwas obtained from all individual participants. The study design received full ethical approval from Department of Neurosurgery, The First People's Hospital of Hangzhou, Hangzhou, China.

\section{Surgical technique}

\section{Repeat microvascular decompression}

Sixty-eight patients who would undergo redo MVD were placed in park bench position, and a typical retrosigmoid cranioectomy was performed at the junction of the transverse and sigmoid sinus. After opening the dura and gentle retraction of the petrosal surface of the cerebellum off the petrous bone, cerebrospinal fluid was slowly released to empty.

In 62 of 68 cases, the thick and adhesional arachnoidal membranes were found obviously and microsurgical sharp dissection, preserving the trigeminal nerve and the corresponding
Table 1. Patient characteristics

\begin{tabular}{lccc}
\hline Factor & $\begin{array}{c}\text { Redo MVD } \\
(\mathbf{n}=68)\end{array}$ & $\begin{array}{c}\text { PBC } \\
(\mathbf{n}=\mathbf{4 2})\end{array}$ & $\boldsymbol{p}$-value \\
\hline Male/female & $23 / 45$ & $15 / 27$ & 0.84 \\
Mean age (years) & 59.3 & 63.4 & 0.32 \\
Right/left & $20 / 48$ & $13 / 29$ & 0.86 \\
Affected trigeminal division & & & \\
V1 & $7(10.3)$ & $5(11.9)$ & 0.13 \\
V2 & $14(20.6)$ & $7(16.7)$ & \\
V3 & $9(13.2)$ & $6(14.3)$ & \\
V1+V2 & $15(22.1)$ & $9(21.4)$ & \\
V2+V3 & $17(28.0)$ & $10(23.8)$ & \\
V1+V2+V3 & $6(8.8)$ & $5(11.9)$ & \\
Mean symptom-free interval & 35.4 & 27.1 & 0.26 \\
(months) & & & \\
\hline
\end{tabular}

$\overline{\text { Values are presented as number (\%). MVD : microvascular decompres- }}$ sion, PBC : percutaneous balloon compression

vessel, was performed. There was no new vascular compression and other abnormal in 61 patients. Then combing the root of trigeminal nerve and replacing the Teflon felt were performed. We found new responsible arteries in five patients and microvascular decompression was carried out. During reoperation new responsible vein, all of which are branches of the rock vein, were found in two patients. Then cutting it by electrocoagulation and the MVD were performed. There were Teflon granuloma in two patients and microsurgical sharp dissection, preserving the trigeminal nerve and the corresponding vessel, with complete removal of the Teflon granuloma was performed. We found the interposed Teflon felt adhering to the trigeminal nerve and had to be carefully dissected from the nerve and conflicting vessels in six patients. In addition, we found that Teflon felt was slipped and the corresponding vessel compressed the root of trigeminal nerve again. And the MVD was performed and Teflon felt was replaced. At last, the dura was accurately closed, and bone was reconstructed.

\section{Percutaneous balloon compression}

The patients were placed in the supine position, with the neck and thorax slightly extended. C-arm image intensifier fluoroscope was used to obtain lateral images and location. The point of entry into the skin was $2.0-3.0 \mathrm{~cm}$ lateral to commissure of the lips. A 14-gage needle which contains a blunt obturator penetrated the foramen ovale under fluoroscopic guid- 
ance. As the blunt obturator was withdrawn, a No. 4 Fogarty balloon catheter, with a fine steel stylet, was advanced into Meckel's cave under direct fluoroscopy. Then, withdrawn the stylet. Next, add undiluted contrast Omnipaque (300 mg iodine $/ \mathrm{mL}$ ) to the balloon until it was proximal to the posterior fossa. Inspect the balloon position and shape depending on the bony landmarks (clivus, sella, and petrous bones), if necessary, the balloon was deflated and the catheter was repositioned until the typical pear shape was achieved. And a pear shape of the balloon indicated sufficient volume. In general, the balloon volume was between 0.4 and $0.8 \mathrm{~mL}$ and balloon compression time was between 4 and 8 minutes. After ganglion compression, the contrast medium was aspirated, the catheter was withdrawn, the puncture site was compressed for 5 minutes.

\section{Follow-up and standard of curative effect}

All patients were contacted 1 month after surgery, then every 6 months thereafter to assess their facial pain outcome. The follow-up was completed by independent supervisor nurse. The mean follow-up time was about 45.6 months postoperatively (ranging from 12 to 72 months). Standard of curative effect : 1) cured : pain completely disappeared after operation. 2) improved : pain did not disappear, but the degree, frequency of the pain was better than preoperation or the scope was smaller. And 3) invalid : there was no significant difference between postoperative pain and preoperative.

\section{Statistical analysis}

Statistical analyses were performed using Statistical Package for the Social Sciences for Windows version 22.0 (SPSS, Chicago, IL, USA). Kaplan-Meier curves were calculated to determine the percentage of patients who were pain free off medications after surgery. Univariate comparisons of continuous variables were compared by use of the Student t-test; proportional differences were compared by the $\chi^{2}$ test.

\section{RESULTS}

There were no significant differences in gender, age, lesion side, affected trigeminal division and symptom-free interval between the two groups ( $p>0.05)$. Comparison of the outcomes for 110 patients who underwent redo MVD or PBC for recurrent trigeminal neuralgia is listed in Table 2. After redo MVD,
65 patients (95.6\%) experienced immediate relief of pain. After PBC, 34 patients (81\%) were immediately relieved of their neuralgia. The differences of the two groups were statistically significant $(p<0.05)$. Follow-up for 1 month after operation. In the redo MVD, 62 patients (91.2\%) were cured, four patients (5.9\%) patients were improved, two patients (2.9\%) were invalid. In the PBC, 30 patients (71.4\%) were cured, nine patients (21.4\%) patients were improved, three patients (7.1\%) were invalid. The clinical effect of the two groups was statistically significant $(p<0.01)$. The detailed results are proved in Table 3. Cured facial pain outcomes were achieved and maintained in 93.4\% and $78.2 \%$ of patients at 1 and 4 years after redo MVD. And $85.1 \%$ and $59.3 \%$ of patients at 1 and 4 years after PBC had cured facial pain outcomes. Mean length of stay was 12.4 days and 10.7 days respectively after redo MVD and PBC. Although mean length of stay after PBC was shorter, the differences were not statistically significant $(p>0.05)$. As far as complications were concerned, After redo MVD, 10 patients (14.7\%) developed herpes simplex on postoperative day 3 , and the symp-

Table 2. Comparison of the outcomes for 110 patients who underwent Redo MVD or PBC for recurrent trigeminal neuralgia

\begin{tabular}{lccc}
\hline Outcomes & Redo MVD & PBC & $p$-value \\
\hline Immediate relief of pain & $65(95.6)$ & $34(81)$ & $<0.05$ \\
Mean length of stay (day) & 12.4 & 10.7 & $>0.05$ \\
\hline Complications & & & \\
Herpes simplex & $10(14.7)$ & $15(35.7)$ & $<0.05$ \\
Facial numbness & $6(8.8)$ & $32(76.2)$ & $<0.05$ \\
Diplopia & 0 & 0 & 0 \\
Keratitis & 0 & 0 & 0 \\
Conjunctivitis & 0 & 0 & 0 \\
Meningitis & 0 & 0 & 0 \\
Hearing loss & 0 & 0 & 0 \\
Annoying dysesthesia & $4(5.9)$ & $9(21.4)$ & $<0.05$ \\
\hline
\end{tabular}

Values are presented as number (\%). MVD : microvascular decompression, $\mathrm{PBC}$ : percutaneous balloon compression

Table 3. Comparison of the clinical effect of two groups in one month after operation

\begin{tabular}{lccc}
\hline Curative effect & Redo MVD & PBC & p-value \\
\hline Cured & $62(91.2)$ & $30(71.4)$ & $<0.01$ \\
Improved & $4(5.9)$ & $9(21.4)$ & $<0.01$ \\
Invalid & $2(2.9)$ & $3(7.1)$ & $<0.01$ \\
\hline
\end{tabular}

Values are presented as number (\%). MVD : microvascular decompression, $\mathrm{PBC}$ : percutaneous balloon compression 
toms lasted for 1 week. Six patients $(8.8 \%)$ occurred facial numbness and all of them recovered after leaving hospital. Four patients (5.9\%) experienced and could tolerate mild to moderate hypoesthesia. No diplopia, keratitis, conjunctivitis, meningitis, hearing loss or death occurred in this study. After PBC, 15 patients $(35.7 \%)$ developed herpes simplex. Facial numbness was the most common complication, affecting 32 patients (76.2\%), but almost all of them could tolerate it well. Nine patients (21.4\%) experienced different degrees of hypoesthesia indicates annoying dysesthesia. None of the patients developed diplopia, keratitis, conjunctivitis, meningitis, hearing loss or death. There were significant differences in herpes simplex, facial numbness and hypoesthesia $(p<0.05)$. In addition to, in PBC procedure 36 patients $(85.7 \%)$ patients achieved the pear shape, three patients (7.1\%) patients achieved the cylindrical shape, and three patients (7.1\%) patients achieved the oval shape $(p<0.01)$.

The symptoms recurred respectively in 15 patients (22.1\%) and 19 patients (45.2\%) after redo MVD and PBC within the entire 6-year follow-up period. The recurrence rate after PBC was higher than that after redo MVD. The Kaplan-Meier evaluation curve is shown in Fig. 1. The differences were statistically significant (log-rank test, $p=0.02$ ).

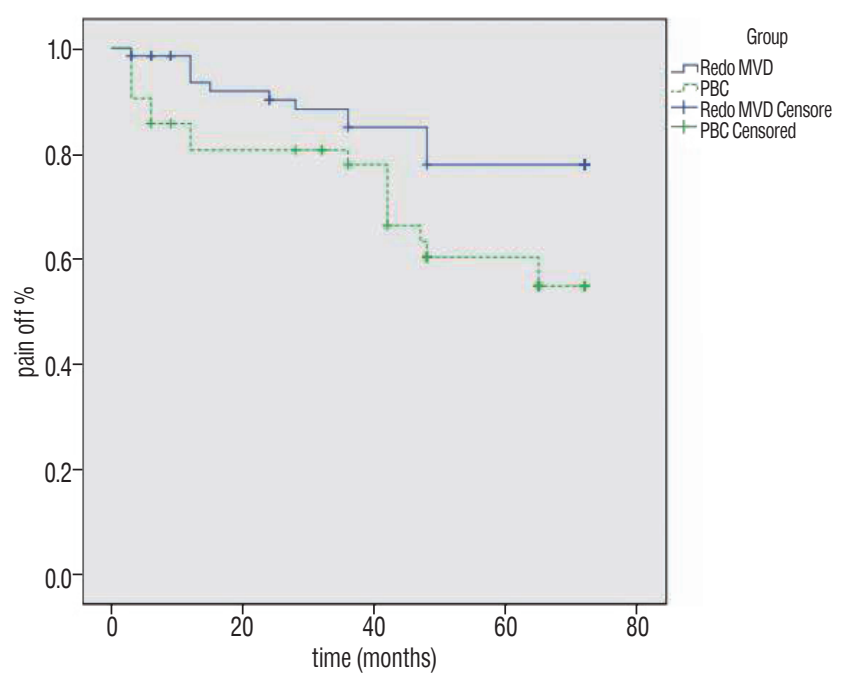

Fig. 1. Kaplan-Meier survival curve comparing the percentage of patients who were pain free off medications after redo MVD (solid line) and PBC (dashed line). Patients having redo MVD were more likely to be pain free after surgery $(p=0.02)$. MVD : microvascular decompression, $\mathrm{PBC}$ : percutaneous balloon compression.

\section{DISCUSSION}

Since Jannetta introduced the microsurgical approach and popularized MVD, MVD has been a major neurosurgical procedure offered to patients with TN when other less invasive treatment options are either no longer helpful or contraindicated, especially when a positive vascular loop abuts on the root entry zone of the trigeminal nerve during pre-operative MRI-TOF brain scan. However, the cure rate is not achieved and maintained in $100 \%$. The rate of recurrence was $17.1 \%$ for a mean follow-up ${\text { period of } 5.4 \text { years }^{18)} \text {, and 25\% recurrence }}^{26)}$ was showed according to Tyler-Kabara's long-term follow-up results of 1185 patients at 5 years ${ }^{26)}$, while Barker et al. ${ }^{3)}$ reported $30 \%$ recurrence at 6.2 years in a large MVD series. The variation in surgical procedure between different institutes, the follow-up period, and the definition of recurrence may lead to differences in recurrent rates.

Many researchers have studied the causes of recurrence. Chen et al. ${ }^{5)}$ reported $50 \%$ operative findings were Teflon granuloma, 30\% operative findings were new arterial loop, 10\% operative findings were venous compression and 10\% operative findings were negative re-exploration. Ugwuanyi and Kitchen ${ }^{27)}$ reported arachnoid adhesions were found in most cases (83.3\%) but usually associated with new vascular loop, neo-arachnoid cyst and sometimes unnamed veins. Jannetta and Bissonette ${ }^{12)}$ re-explored 51 cases of recurrent TN after MVD, and 42 cases (82\%) were vascular loops. Kureshi and Wilkins ${ }^{14)}$ found only $30 \%$ of cases were a new neurovascular compression and most of these were scarred implants, re-exploring 23 cases of recurrent TN after their prior MVD. The procedure for the first MVD should significantly influence the causes of recurrence. For the recurrent TN patients after MVD, they were examined by preoperative MRI-TOF brain scan in this study. As one of selection criteria, although its specificity and sensitivity was not $100 \%$, it was applied to the clinic widely due to the high cure rate after MVD when a positive vascular loop abuts on the root entry zone of the trigeminal nerve.

Some clinicians chose redo MVD. In the report of Yang et al. $^{29)}$, the pain of 14 cases (93.3\%) was completely relieved and only one case $(6.7 \%)$ was partially relieved with a mean followup of 3.2 years, and no patient experienced recurrence. In their mean 55.4 months follow-up of 12 patients, Gu and Zhao ${ }^{9)}$ reported eight patients (66.7\%) had a BNI score of I, two patients (16.7\%) had a BNI score of II, and two patients (16.7\%) had a BNI score of III, of which one patient suffered from transient 
psychiatric symptoms and one patient developed permanent annoying dysesthesia. Lee et al. ${ }^{15)}$ reported the results of second MVD, $81.3 \%$ of the cases were improved, but only eight patients (25\%) experienced complete pain relief. However, some clinicians $^{6}$ chose $\mathrm{PBC}$ in consideration of the high risk of morbidity after redo MVD or a case in which a young patient expects mild or moderate numbness after a percutaneous operation. Du et al. ${ }^{8)}$ reported $43(82.7 \%)$ patients remained pain-free, four patients (7.7\%) had mild recurrence, and three patients (5.8\%) had severe recurrence, with a mean follow-up time of 37.6 months after surgery. Park et al. ${ }^{21)}$ reported the immediate pain relief rate was $92 \%$, and the recurrence rate was $16 \%$ with a mean followup period of 3.5 years. As an effective measure after MVD, $\mathrm{PBC}$ is also the main way to treat $\mathrm{TN}$ recurrence, which is widely accepted by patients due to low risky, small trauma, short operation and low cost.

As two effective treatment for the patients whose first MVD was failed, they are popular in the world and also commonly used in our center, which treated thousands of TN recurrence. Nevertheless, which treatment method is better? A large number of related literatures usually contain the varying, often conflicting, conclusions and remarkably few randomized controlled trials, which represent the best form of evidence. Patients who are increasingly well informed wish to take part in the decision-making process and regard which treatment is more appropriate $^{16)}$. As a convenient and reliable research method, prospective cohort study avoids subjective bias from the known prognostic, compared with the retrospective study. Moreover, unlike randomized controlled trials, it does not need enough extreme eligible samples and does not have the problem of lager expenditure and ethics and morals.

A prospective trial is performed in this study. The two groups were similar with regard to sex, age, lesion side, pain location, and symptom-free interval. Sixty-five patients (95.6\%) undergoing repeat MVD achieved immediate relief of pain, and it was higher compared with the patients undergoing PBC (81\%). And this is the main reason that the patients are more inclined to undergo repeat MVD when TN recurrence occurs. In the redo MVD group, $91.2 \%$ of intraoperative findings were the thick and adhesional arachnoidal membranes and only seven patients (10.3\%) were found new vascular compression. Therefore, MVD combined with neurocombing has been applied to these patients. Facial numbness was the most common postoperative complications of $\mathrm{PBC}$, affecting 32 patients $(76.2 \%)$. This might be related to longer compression time. Skirving and $\operatorname{Dan}^{24)}$ reported facial numbness morbidity was $89 \%$ (441 patients), and the compression time was between 2 and 7 minutes. Stomal-Słowińska et al. ${ }^{25}$ reported most patients (90\%) experienced facial numbness after surgery, and the inflated balloon lasted for 1-1.5 minutes. However, for TN recurrent patients, longer compression time usually is necessary and linked closely with clinical effect. Recently it has also been shown that the pear shape is the most important factor related to obtaining a satisfying result, and most authors have stressed to obtain a pear shape of the inflated balloon, which usually brings the operator some challenge ${ }^{4)}$. Though mean length of stay in redo MVD group was longer than in $\mathrm{PBC}$ group and the trauma was larger, complications including herpes simplex, facial numbness, and dysesthesia were less and immediate relief rate of pain and clinical effect in one month after operation were better. In addition, the recurrence rate after PBC (45.2\%) was higher than that after redo MVD (22.1\%), according to the Kaplan-Meier evaluation curve. As for other complication, they were not found in two groups in this study.

\section{CONCLUSION}

A prospective comparison of patients with $\mathrm{TN}$ recurrence found that redo MVD was a more effective procedure than PBC. Both the cure rate and immediate relief of pain were better, and the incidence of complications was lower after redo MVD. Further study is needed to better understand the relative effectiveness of PBC to glycerol rhizotomy, radiofrequency rhizotomy, and stereotactic radiosurgery for patients with $\mathrm{TN}$ recurrence.

\section{CONFLICTS OF INTEREST}

No potential conflict of interest relevant to this article was reported.

\section{INFORMED CONSENT}

Informed consent was obtained from all individual participants included in this study. 


\section{References}

1. Amador N, Pollock BE : Repeat posterior fossa exploration for patients with persistent or recurrent idiopathic trigeminal neuralgia. J Neurosurg 108 : 916-920, 2008

2. Bakker NA, Van Dijk JM, Immenga S, Wagemakers M, Metzemaekers JD : Repeat microvascular decompression for recurrent idiopathic trigeminal neuralgia. J Neurosurg 121 : 936-939, 2014

3. Barker FG 2nd, Jannetta PJ, Bissonette DJ, Larkins MV, Jho HD : The longterm outcome of microvascular decompression for trigeminal neuralgia. New Engl J Med 334 : 1077-1083, 1996

4. Bergenheim AT, Asplund $P$, Linderoth $B$ : Percutaneous retrogasserian balloon compression for trigeminal neuralgia: review of critical technical details and outcomes. World Neurosurg 79 : 359-368, 2013

5. Chen J, Lee S, Lui T, Yeh Y, Chen T, Tzaan W : Teflon granuloma after microvascular decompression for trigeminal neuralgia. Surg Neurol 53 : 281 287, 2000

6. Chen JF, Tu PH, Lee ST : Repeated percutaneous balloon compression for recurrent trigeminal neuralgia: a long-term study. World Neurosurg 77 : 352-356, 2012

7. Cho DY, Chang CG, Wang YC, Wang FH, Shen CC, Yang DY : Repeat operations in failed microvascular decompression for trigeminal neuralgia. Neurosurgery 35 : 665-669; discussion 669-670, 1994

8. Du Y, Yang D, Dong X, Du Q, Wang H, Yu W : Percutaneous balloon compression $(\mathrm{PBC})$ of trigeminal ganglion for recurrent trigeminal neuralgia after microvascular decompression (MVD). Ir J Med Sci 184 : 745-751, 2015

9. Gu W, Zhao W: Microvascular decompression for recurrent trigeminal neuralgia. J Clin Neurosci 21 : 1549-1553, 2014

10. Jannetta PJ : Arterial compression of the trigeminal nerve at the pons in patients with trigeminal neuralgia. J Neurosurg 26 Suppl : 159-162, 1967

11. Jannetta PJ : Neurovascular compression in cranial nerve and systemic disease. Ann Surg 192 : 518-525, 1980

12. Jannetta PJ, Bissonette DJ : Management of the failed patient with trigeminal neuralgia. Clin Neurosurg 32 : 334-347, 1985

13. Jannetta PJ, Tew JM Jr : Treatment of trigeminal neuralgia. Neurosurgery 4 : 93-94, 1979

14. Kureshi SA, Wilkins RH : Posterior fossa reexploration for persistent or recurrent trigeminal neuralgia or hemifacial spasm: surgical findings and therapeutic implications. Neurosurgery 43 : 1111-1117, 1998

15. Lee SH, Levy El, Scarrow AM, Kassam A, Jannetta PJ : Recurrent trigeminal neuralgia attributable to veins after microvascular decompression.

Neurosurgery 46 : 356-361; discussion 361-362, 2000

16. Lopez BC, Hamlyn PJ, Zakrzewska JM : Systematic review of ablative neurosurgical techniques for the treatment of trigeminal neuralgia. Neurosurgery 54 : 973-982; discussion 982-983, 2004

17. Massager $N$, Lorenzoni J, Devriendt $D$, Desmedt $F$, Brotchi J, Levivier $M$ :
Gamma knife surgery for idiopathic trigeminal neuralgia performed using a far-anterior cisternal target and a high dose of radiation. J Neurosurg $100:$ 597-605, 2004

18. Matsushima T, Yamaguchi T, Inoue TK, Matsukado K, Fukui M : Recurrent trigeminal neuralgia after microvascular decompression using an interposing technique. Teflon felt adhesion and the sling retraction technique. Acta Neurochir (Wien) 142 : 557-561, 2000

19. Meybodi AT, Habibi Z, Miri M, Tabatabaie SA : Microvascular decompression for trigeminal neuralgia using the 'Stitched Sling Retraction' technique in recurrent cases after previous microvascular decompression. Acta Neurochir 156 : 1181-1187; discussion 1187, 2014

20. Nanda A, Javalkar V, Zhang S, Ahmed 0 : Long term efficacy and patient satisfaction of microvascular decompression and gamma knife radiosurgery for trigeminal neuralgia. J Clin Neurosci 22 : 818-822, 2015

21. Park SS, Lee MK, Kim JW, Jung JY, Kim IS, Ghang CG : Percutaneous balloon compression of trigeminal ganglion for the treatment of idiopathic trigeminal neuralgia : experience in 50 patients. J Korean Neurosurg Soc 43 : 186-189, 2008

22. Premsagar IC, Moss T, Coakham HB : Teflon-induced granuloma following treatment of trigeminal neuralgia by microvascular decompression. report of two cases. J Neurosurg 87 : 454-457, 1997

23. Rzaev DA, Kulikova EV, Moysak Gl, Voronina El, Ageeva TA : Teflon granuloma after microvascular decompression of the trigeminal nerve root in a patient with recurrent trigeminal neuralgia. Zh Vopr Neirokhir Im N N Burdenko 80 : 78-83, 2016

24. Skirving DJ, Dan NG : A 20-year review of percutaneous balloon compression of the trigeminal ganglion. J Neurosurg 94 : 913-917, 2001

25. Stomal-Słowińska M, Słowiński J, Lee TK, Uitti RJ, Deen HG, Reimer R, et al. : Correlation of clinical findings and results of percutaneous balloon compression for patients with trigeminal neuralgia. Clin Neurol Neurosurg $113: 14-21,2011$

26. Tyler-Kabara EC, Kassam AB, Horowitz MH, Urgo L, Hadjipanayis C, Levy $\mathrm{El}$, et al. : Predictors of outcome in surgically managed patients with typical and atypical trigeminal neuralgia: comparison of results following microvascular decompression. J Neurosurg 96 : 527-531, 2002

27. Ugwuanyi UC, Kitchen ND : The operative findings in re-do microvascular decompression for recurrent trigeminal neuralgia. Br J Neurosurg 24 : 26-30, 2010

28. Wei Y, Pu C, Li N, Cai Y, Shang H, Zhao W : Long-term therapeutic effect of microvascular decompression for trigeminal neuralgia: Kaplan-Meier analysis in a consecutive series of 425 patients. Turk Neurosurg 28 : 88 93, 2018

29. Yang DB, Jiang DY, Chen HC, Wang ZM : Second microvascular decompression for trigeminal neuralgia in recurrent cases after microvascular decompression. J Craniofac Surg 26 : 491-494, 2015

30. Zhang H, Lei D, You C, Mao BY, Wu B, Fang Y : The long-term outcome predictors of pure microvascular decompression for primary trigeminal neuralgia. World Neurosurg 79 : 756-762, 2013 